\title{
Distinguishing trees in linear time
}

\author{
Antoni Lozano* \\ Dpt. Llenguatges i Sistemes Informàtics \\ Universitat Politècnica de Catalunya \\ Barcelona, Spain \\ antoni@lsi.upc.edu
}

\author{
Mercè Mora ${ }^{\dagger}$ Carlos Seara \\ Dpt. Matemàtica Aplicada II \\ Universitat Politècnica de Catalunya \\ Barcelona, Spain \\ \{merce.mora, carlos.seara\}@upc.edu
}

Submitted: Apr 15, 2011; Accepted: Apr 26, 2012; Published: May 21, 2012

Mathematics Subject Classifications: 05C85, 68R05, 05C15, 05C78

\begin{abstract}
A graph is said to be $d$-distinguishable if there exists a $d$-labeling of its vertices which is only preserved by the identity map. The distinguishing number of a graph $G$ is the smallest number $d$ for which $G$ is $d$-distinguishable. We show that the distinguishing number of trees and forests can be computed in linear time, improving the previously known $\mathcal{O}(n \log n)$ time algorithm.
\end{abstract}

\section{Introduction}

Let $G$ be a connected graph with $n$ vertices $^{1}$. A $d$-labeling of $G$ is a total function $\phi: V(G) \longrightarrow\{1,2, \ldots, d\}$. We say that $\phi$ distinguishes $G$ if $G$ has no label-preserving automorphism different from the identity map. In this case, we say that $\phi$ is a distinguishing d-labeling of $G$. Such a labeling is said to break or destroy the symmetries of $G$. The distinguishing number of $G, D(G)$, is the minimum number $d$ of labels needed so that $G$ has a distinguishing $d$-labeling. A graph $G$ having a distinguishing $d$-labeling is said to be $d$-distinguishable.

Distinguishing numbers were first introduced by Albertson and Collins [2]. The parameter can be thought of as a measure of the symmetry of a graph, i.e., if $G$ and $G^{\prime}$

*Antoni Lozano is supported by projects MTM2011-28800-C02-01 and BASMATI, Biological and Social Mining: Algorithms, Theory, and Implementation TIN2011-27479-C04-03.

${ }^{\dagger}$ Mercè Mora and Carlos Seara are partially supported by projects MTM2009-07242, Gen Cat DGR2009GR1040, and the ESF EUROCORES programme EuroGIGA-ComPoSe IP04-MICINN Project EUI-EURC-2011-4306.

${ }^{1}$ Graphs in this paper are finite, undirected and simple. The vertex-set and the edge-set of a graph $G$ are denoted by $V(G)$ and $E(G)$, respectively. The order of $G$ is the number of its vertices, denoted by $|V(G)|$. For more terminology we follow [19]. 
have the same number of vertices but $D(G)>D\left(G^{\prime}\right)$, then $G$ is more symmetric than $G^{\prime}$ because more colors are needed to destroy its automorphisms than those of $G^{\prime}$.

It is not known if the problem of computing $D(G)$ is polynomially-time solvable or NP-hard. Russell and Sundaram [17] showed that determining if $D(G)>d$ belongs to the class AM, i.e., the set of languages for which there are Arthur-Merlin games. However, when $G$ is restricted to certain graph families such as cycles, hypercubes, acyclic graphs, and planar graphs, the problem can be solved efficiently $[2,4,5,6,7,10]$. See $[1,5,8,9$, $11,14,15,16,18]$ for other works on distinguishing number problems.

For the computation of the distinguishing number of trees and forests with $n$ vertices, Cheng [10] and Arvind and Devanur [4] presented an $\mathcal{O}(n \log n)$ time algorithm which uses a binary search to compute the distinguishing number of a tree. Improving this time complexity was our main motivation for the design of an optimal linear-time algorithm for computing the distinguishing number of trees and forests, and this is the main result of our paper.

In Section 2 we will focus on the design of a linear time algorithm for rooted trees which is based on properties proved by Cheng [10] and follow her notation whenever possible. As a consequence of our result, we show in Section 3 that there are linear-time algorithms for computing the distinguishing numbers of trees and forests. Finally, in Section 4 we conjecture logarithmic factor improvements for other graph classes.

\section{Distinguishing Rooted Trees}

\subsection{Preliminaries}

We start with some notation. By $\operatorname{Aut}(G)$ we denote the automorphism group of a graph $G$. As usual, two graphs $G$ and $H$ are isomorphic, denoted by $G \cong H$, if there is a permutation $\pi: V(G) \rightarrow V(H)$ which preserves adjacencies, that is, $\{u, v\} \in E(G)$ if and only if $\{\pi(u), \pi(v)\} \in E(H)$ for any $u, v \in V(G)$.

Given a graph $G$ and a labeling $\phi$ of $G$, we represent the corresponding labeled graph by $(G, \phi)$. In this case, $\operatorname{Aut}(G, \phi)$ consists of all the automorphisms of $\operatorname{Aut}(G)$ which preserve the labeling $\phi$, that is, $\pi \in \operatorname{Aut}(G, \phi)$ if and only if $\phi \in \operatorname{Aut}(G)$ and $\phi(v)=\phi(\pi(v))$. We also consider the extension of isomorphism to labeled graphs. Given two labeled graphs $(G, \phi)$ and $(H, \varphi)$, we say that they are isomorphic if there is a permutation $\pi: V(G) \rightarrow V(H)$ which preserves adjacencies as defined above, but also preserves labels, that is, $\phi(v)=\varphi(\pi(v))$ for each $v \in V(G)$. In this case, we write $(G, \phi) \cong(H, \varphi)$. Two distinguishing $d$-labelings $\phi$ and $\varphi$ of a graph $G$ are said to be equivalent if $(G, \phi) \cong(G, \varphi)$.

Given a rooted tree $T$, we denote its root by $r(T)$. We also denote by $T_{u}$ the subtree of $T$ rooted at vertex $u$ of $T$, and we call components of $T$ to all the subtrees $T_{u}$ of $T$ where $u$ is a child of $r(T)$. Any isomorphism between two rooted trees $T_{1}$ and $T_{2}$ must map $r\left(T_{1}\right)$ into $r\left(T_{2}\right)$. In the same way, any automorphism of a rooted tree must map the root into itself.

As we will see, the distinguishing number of a rooted tree can be computed using a recursive formula. Call $D(T, d)$ to the number of inequivalent distinguishing $d$-labelings 
of a rooted tree $T$. For instance, if $T$ is a rooted tree consisting of a single path of $k$ vertices, then $D(T, d)=d^{k}$, but if $T$ is a full binary tree of (any) depth $k$, then $D(T, 2)$ is just 2 since we can assign two possible labels to the root while the rest of the tree has a unique distinguishing 2-labeling up to isomorphism. Clearly, for any graph $G$,

$$
D(G)=\min \{d \mid D(G, d)>0\}
$$

and, therefore, computing $D(T, d)$ for any $d$ is all that is needed to compute $D(T)$ for a rooted tree $T$. We also observe the following relation between $D(T)$ and $D(T, d)$.

Proposition 1. For any rooted tree $T$ and for any $d \geqslant D(T)$, it holds $D(T, d) \geqslant d$.

Proof. For any $d \geqslant D(T)$, the rooted tree $T$ is clearly $d$-distinguishable, so suppose $\phi$ is some $d$-distinguishing labeling of $T$. Changing the label assigned by $\phi$ to the root gives $d$ inequivalent labelings of $T$, that is,

$$
\phi_{i}(u)= \begin{cases}i, & \text { if } u=r(T) \\ \phi(u), & \text { if } u \neq r(T) .\end{cases}
$$

for every $i \in\{1, \ldots, d\}$. To see that the labelings are inequivalent, suppose on the contrary that two such labelings, say $\phi_{i}$ and $\phi_{j}$ (where $1 \leqslant i<j \leqslant d$ ), are equivalent. Then, there would be a mapping $\pi$ between the labeled copies $\left(T, \phi_{i}\right)$ and $\left(T, \phi_{j}\right)$ such that $\phi_{i}(r(T))=$ $\phi_{j}(\pi(r(T)))=\phi_{j}(r(T))$, where the last equality holds because any isomorphism between labeled copies of a rooted tree must map the root to itself. But then, by definition of $\phi_{i}$ and $\phi_{j}$, we get $i=j$, contradicting our assumption that $i<j$.

The existence of the inequivalent $d$-labelings $\phi_{1}, \ldots, \phi_{d}$ for $T$ implies that $D(T, d) \geqslant d$.

Now, we consider the recursive formula developed in [4] and [10] which counts the number of inequivalent distinguishing $d$-labelings of a rooted tree, and how to derive the distinguishing number from it in two ways.

Proposition 2. ([10], Th. 3.2, Cor. 3.3, Th. 4.2) Let $T$ be a rooted tree and $\mathcal{T}$ be the set of the components of $T$. Suppose that $\mathcal{T}$ has exactly $g$ distinct isomorphism classes of trees where the ith isomorphism class consists of $m_{i}$ copies of the rooted tree $T_{u_{i}}$; i.e., $\mathcal{T}=m_{1} T_{u_{1}} \cup m_{2} T_{u_{2}} \cup \cdots \cup m_{g} T_{u_{g}}$. Then,

1. $D(T, d)=d \prod_{i=1}^{g}\left(\begin{array}{c}D\left(T_{u_{i}}, d\right) \\ m_{i}\end{array}\right)$.

2. $D(T)=\min \left\{d \mid \forall i \in\{1, \ldots, g\} \quad D\left(T_{u_{i}}, d\right) \geqslant m_{i}\right\}$.

3. $D(T)=\max \left\{\min \left\{d \mid D\left(T_{u_{i}}, d\right) \geqslant m_{i}\right\} \mid 1 \leqslant i \leqslant g\right\}$.

By Proposition 2(1), in order to compute $D(T)$ for a rooted tree $T$, it is enough to know a list of values $\left\{\left(m_{1}, u_{1}\right), \ldots,\left(m_{g}, u_{g}\right)\right\}$, where the degree of $r(T)$ equals $\sum_{i=1}^{g} m_{i}$ and for each pair $\left(m_{i}, u_{i}\right), u_{i}$ is a child of $r(T)$ and $m_{i}$ is the multiplicity of the isomorphic copies of $T_{u_{i}}$ which appear as components of $T$. We take advantage of the fact that Cheng [10] shows 
a method to compute exactly this information. We will assume that a new procedure called Compute-LIST, given a rooted tree $T$, returns the list $\left\{\left(m_{1}, u_{1}\right), \ldots,\left(m_{g}, u_{g}\right)\right\}$ defined above (in [10], this can be accomplished by calling procedure FIND-ISOMORPH, then ESSENTIAL and, finally, taking the first output). Cheng [10] shows that this can be done in linear time.

\subsection{Linear Time Algorithm}

We will describe the procedures used in our main algorithm. In the first place, procedure Colorings $(T, d)$, given a rooted tree $T$ and a constant $d$, computes $D(T, d)$ in linear time. We will not detail the algorithm here since it is already described by Cheng [10] and by Arvind and Devanur [4]. This procedure is called EVALUATE in [10] and Inequiv in $[4]$.

Note that, according to Proposition 2(2), given a rooted tree $T$ of order $n, D(T)$ can already be computed by making calls to $\operatorname{ColoRings}(T, d)$ for different values of $d$, $1 \leqslant d \leqslant n-1$. Since each call takes linear time, using binary search in $d$, it is possible to find $D(T)$ in time $\mathcal{O}(n \log n)$, as it is argued in [4] and [10]. This is precisely the common method in $[4,10]$ which works in time $\mathcal{O}(n \log n)$. In order to lower it to an overall linear time, we need to carefully call this procedure for the subtrees of $T$ only when it is needed. To do so, we need the information contained in the list $\left\{\left(m_{1}, u_{1}\right), \ldots,\left(m_{g}, u_{g}\right)\right\}$, which will be obtained in linear time by calling to a procedure COMPUTE-LIST, as stated at the end of Subsection 2.1. Our algorithm is the following (see Figure 1 for an example).

\section{Distinguishing $(T)$}

Input: A rooted tree $T$ with $n$ vertices

Output: $D(T)$

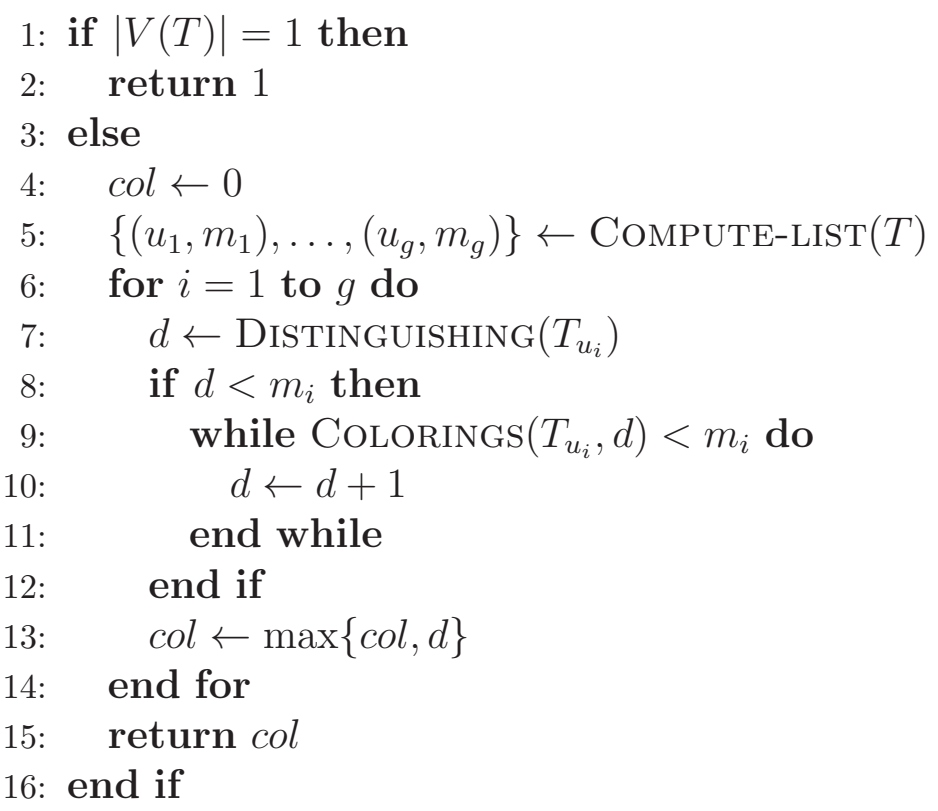




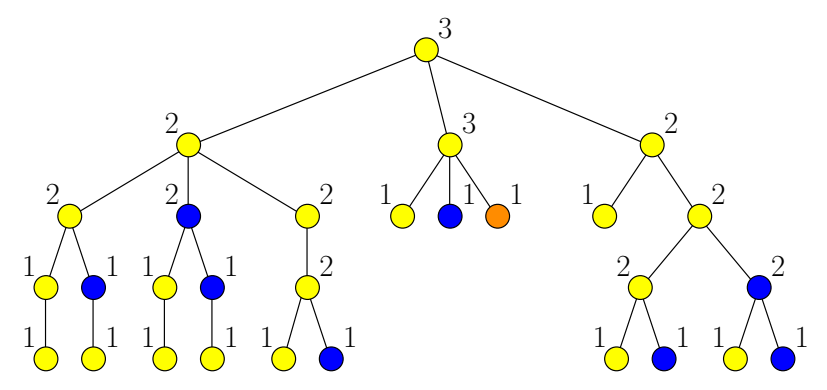

Figure 1: Each vertex is labeled with the distinguishing number of the rooted subtree it defines.

Theorem 3. (Correctness) Given a rooted tree $T$ as input, procedure Distinguishing returns $D(T)$.

Proof. We show it by induction on the order of $T$. If the rooted tree $T$ has only one vertex, Distinguishing returns 1 at line 2, which is correct. Suppose now that the order of $T$ is $n>1$. For each $i$, procedure Distinguishing makes a recursive call on the subtree $T_{u_{i}}$ in line 7. By induction hypothesis, we can assume that the result is $d=D\left(T_{u_{i}}\right)$. Now we can distinguish two cases:

1. If $d \geqslant m_{i}$, then by Proposition $1, D\left(T_{u_{i}}, d\right) \geqslant d \geqslant m_{i}$.

2. If $d<m_{i}$, then after the while loop in lines $9-11, d$ is the smallest value satisfying $D\left(T_{u_{i}}, d\right) \geqslant m_{i}$.

In any case, the value of $d$ at line 13 for a given $i$ is the smallest value such that $D\left(T_{u_{i}}, d\right) \geqslant$ $m_{i}$. Since the value col returned by Distinguishing is the maximum of such values for $1 \leqslant i \leqslant g$, it must equal $D(T)$ by Proposition $2(3)$.

Theorem 4. (Complexity) Procedure Distinguishing works in $\mathcal{O}(n)$ time.

Proof. Let $T$ be the rooted tree of order $n$ given as input and let $T_{u_{1}}, \ldots, T_{u_{g}}$ be its different components up to isomorphism, with orders $n_{1}, \ldots, n_{g}$, respectively (In [3] the authors provided a linear time isomorphism test for trees). Note that since $m_{i}$ is the number of isomorphic copies for $T_{u_{i}}$, it holds that

$$
n=1+\sum_{i=1}^{g} m_{i} \cdot n_{i} .
$$

Since line 5 takes linear time, let $a$ stand for a constant such that an bounds the time required by procedure Distinguishing to execute lines 1-5 and return col at line 15 . Additionally, the for loop between lines 6-14 does some work in constant time, say $b$, plus a maximum of $m_{i}$ calls to $\operatorname{Colorings}\left(T_{u_{i}}, d\right)$. The reason why Colorings is called at most $m_{i}$ times for a given $i$ is that the while loop in lines 9-11 is never executed for $d=m_{i}$ since, by Proposition $1, D\left(T_{u_{i}}, m_{i}\right) \geqslant m_{i}$ and the condition of the loop would not 
hold. Now, since Colorings works in linear time, we can bound the overall work done between lines 9 and 11 by $c n_{i}$ for a constant $c$. Now, if we denote the running time of Distinguishing by $R(n)$, we have

$$
R(n) \leqslant a n+\sum_{i=1}^{g}\left(b+c\left(m_{i}-1\right) n_{i}+R\left(n_{i}\right)\right) .
$$

Let $e$ be a constant such that $e \geqslant a+b+c$. We will show by induction that the running time of Distinguishing $(T)$ is bounded by an $+e(n-1)$. For $n=1$, we have $R(n) \leqslant a=a n+e(n-1)$. In the general case $n>1$, we unfold the summation in Equation (2) and get:

$$
R(n) \leqslant a n+b g+c \sum_{i=1}^{g}\left(\left(m_{i}-1\right) n_{i}\right)+\sum_{i=1}^{g}\left(a n_{i}+e\left(n_{i}-1\right)\right),
$$

where the last term comes from the induction hypothesis. Now,

$$
\begin{aligned}
R(n) & \leqslant a n+b g+(e-a) \sum_{i=1}^{g}\left(\left(m_{i}-1\right) n_{i}\right)+a \sum_{i=1}^{g} n_{i}-e g+e \sum_{i=1}^{g} n_{i} \\
& \leqslant a n+b g+-a \sum_{i=1}^{g}\left(\left(m_{i}-1\right) n_{i}\right)+a \sum_{i=1}^{g} n_{i}-e g+e(n-1) \\
& \leqslant a n-a \sum_{i=1}^{g}\left(m_{i}-1\right) n_{i}+a \sum_{i=1}^{g} n_{i}+e(n-1) \\
& \leqslant a n-a(n-1)+a \sum_{i=1}^{g} n_{i}+e(n-1) \\
& =a\left(1+\sum_{i=1}^{g} n_{i}\right)+e(n-1) \leqslant a n+e(n-1)
\end{aligned}
$$

where both Equation (1) and the fact that $e \geqslant a+b+c$ have been used. Therefore, we conclude that $R(n)$ is $\mathcal{O}(n)$.

\section{Distinguishing Trees and Forests}

There is an easy way to transform the problem of computing $D(T)$ for a general tree $T$ into the one of computing $D\left(T^{\prime}\right)$ for a rooted tree $T^{\prime}$. This can be done using the concept of tree center, as is done in [4] and [10]. A center of a tree $T$ is a vertex $v$ such that the maximal distance of $v$ to the other vertices is minimized. It is well known that every tree has either one center or two adjacent centers. In the first case, the tree can already be considered a rooted tree with root at its center, while in the second case, a new vertex can be inserted between the centers and then used as its root. As mentioned in [4] and [10], this transformation can be done in linear time. 
Proposition 5. Given a tree $T$, it is possible to compute a rooted tree $T^{\prime}$ in linear time such that $D(T)=D\left(T^{\prime}\right)$.

As a direct consequence of Proposition 5 and Theorems 3 and 4 , we conclude the following.

Corollary 6. The distinguishing number of a tree with $n$ vertices can be computed in $\mathcal{O}(n)$ time.

In the case of forests, we use the transformation from Cheng's paper [10], which is as follows. Suppose that $F$ is a forest and define the following trees $T_{1}$ and $T_{2}$. In the first place, create two vertices $v_{1}$, and $v_{2}$ which will act as the respective roots of $T_{1}$ and $T_{2}$, respectively. In the second place, transform each connected component of $F$ into a rooted tree as indicated before Proposition 5. Since this can be done in two ways, depending on whether the original tree is unicentral or bicentral, call $F_{1}\left(F_{2}\right)$ to the set of rooted trees obtained from the unicentral (bicentral) trees in $F$ in the way indicated before Proposition 5 . Finally, join $v_{j}$ to the roots of all trees in $F_{j}$, for $1 \leqslant j \leqslant 2$. We can now state the following regarding the above construction.

Proposition 7. Given a forest $F$, it is possible to compute two rooted trees $T_{1}$ and $T_{2}$ in linear time such that $D(F)=\max \left\{D\left(T_{1}\right), D\left(T_{2}\right)\right\}$.

Proof. It is well known that the centers of a tree can be computed in linear time. So, given a forest $F$ with $n$ vertices, the above transformation into trees $T_{1}$ and $T_{2}$ can be done in time $\mathcal{O}(n)$. Moreover, since no automorphism of $F$ can map a unicentral tree into a bicentral tree or viceversa, nontrivial automorphisms of $F$ induce separate nontrivial automorphisms in $T_{1}$ and $T_{2}$. Suppose that $d=\max \left\{D\left(T_{1}\right), D\left(T_{2}\right)\right\}$. Then, $d$ labels are enough to break symmetries in both $T_{1}$ and $T_{2}$, that is, there must be two $d$-labelings $\phi_{1}, \phi_{2}$ such that both $\operatorname{Aut}\left(T_{1}, \phi_{1}\right)$ and $\operatorname{Aut}\left(T_{2}, \phi_{2}\right)$ are trivial. Then, one can define a distinguishing $d$-labeling $\phi$ of $F$ : Given a vertex $u$ of $F$, if $u \in T_{1}$, set $\phi(u)=\varphi_{1}(u)$ and, otherwise, set $\phi(u)=\varphi_{2}(u)$. In case $\operatorname{Aut}(F, \phi)$ was nontrivial, then one of $\operatorname{Aut}\left(T_{1}, \varphi_{1}\right)$ or $\operatorname{Aut}\left(T_{2}, \varphi_{2}\right)$ would be nontrivial. Thus, $\operatorname{Aut}(F, \phi)$ must be trivial, and $D(F) \leqslant d$. But we can discard the case when $D(F)=d^{\prime}<d$ since it would make it possible to use the distinguishing $d^{\prime}$-labeling of $F$ to define a distinguishing $d^{\prime}$-labeling of $T_{1}$ and $T_{2}$, contradicting the definition of $d$ as the maximum between $D\left(T_{1}\right)$ and $D\left(T_{2}\right)$. Therefore, $D(F)=\max \left\{D\left(T_{1}\right), D\left(T_{2}\right)\right\}$.

Now, it is clear that our algorithm Distinguishing from Section 2 can be used twice in combination with Proposition 7 to yield the following result.

Corollary 8. The distinguishing number of a forest with $n$ vertices can be computed in $\mathcal{O}(n)$ time. 


\section{Conclusions and applications}

We have shown that the distinguishing number of trees and forests can be computed in linear time, improving the previously known $\mathcal{O}(n \log n)$ time algorithm. We believe that our algorithmic technique in Section 2 can be applied to improve by a logarithmic factor (caused by a binary search in the last step of the algorithms) the complexities of computing distinguishing numbers and distinguishing chromatic numbers of the following graph classes: (1) the distinguishing number of (i) planar graphs computed by Arvind et al. $[4,5]$ and (ii) interval graphs computed by Cheng [11]; (2) the distinguishing chromatic number (due to Collins and Trenk [12], see also [13]) of: (i) trees computed by Cheng [11] and (ii) interval graphs computed by Cheng [11].

\section{References}

[1] M. O. Albertson. Distinguishing cartesian powers of graphs. Electron. J. Combin., Vol. 12, N17, 2005.

[2] M. O. Albertson and K. Collins. Symmetry breaking in graphs. Electron. J. Combin., Vol. 3, R18, 1996.

[3] A. V. Aho, J. E. Hopcroft, and J. D. Ullman. The design and analysis of computer algorithms. Addison-Wesley Pub. Co. 1974.

[4] V. Arvind and N. R. Devanur. Symmetry breaking in trees and planar graphs by vertex coloring. Proceedings of the Nordic Combinatorial Conference, 2004.

[5] V. Arvind, C. T. Cheng, and N. R. Devanur. On computing the distinguishing numbers of planar graphs and beyond: a counting approach. SIAM Journal on Discrete Mathematics, 22:4, pp. 1297-1324, 2008.

[6] B. Bogstad and L. J. Cowen. The distinguishing number of the hypercube. Discrete Math., Vol. 283, No. 1-3, pp. 29-35, 2004.

[7] M. Chan. The distinguishing number of the augmented cube and hypercube powers. Discrete Math., Vol. 308, pp. 2330-2336, 2008.

[8] M. Chan. The distinguishing number of the direct product and wreath product action. Journal of Algebraic Combinatorics, Vol. 24, pp. 331-345, 2006.

[9] M. Chan. The maximum distinguishing number of a group. Electron. J. Combin., Vol. 13, R70, 2006.

[10] C. Cheng. On computing the distinguishing numbers of trees and forests. Electron. J. Combin., Vol. 13, R11, 2006.

[11] C. Cheng. On computing the distinguishing and distinguishing chromatic numbers of interval graphs and other results. Discrete Math., 309:16, pp. 5169-5182, 2009.

[12] K. L. Collins and A. N. Trenk. The distinguishing chromatic number. Electron. J. Combin., Vol. 13, R16, 2006. 
[13] E. M. Eschen, C. T. Hoàng, R. Sritharan, and L. Stewart. On the complexity of deciding whether the distinguishing chromatic number of a graph is at most two. Discrete Math., Vol. 311, Issue 6, pp. 431-434, 2011.

[14] W. Imrich and S. Klavžar. Distinguishing cartesian powers of graphs. Journal of Graph Theory, Vol. 53, Issue 3, pp. 250-260, 2006.

[15] W. Imrich, J. Jerebicb, and S. Klavžar. The distinguishing number of cartesian products of complete graphs. European J. Combin., Vol. 29, Issue 4, pp. 922-929, 2008.

[16] S. Klavžar and X. Zhu. Cartesian powers of graphs can be distinguished by two labels. European J. Combin., Vol. 28, pp. 303-310, 2007.

[17] A. Russell and R. Sundaram. A note on the asymptotics and computational complexity of graph distinguishability. Electron. J. Combin., Vol. 5, R23, 1998.

[18] J. Tymoczko. Distinguishing numbers for graphs and groups. Electron. J. Combin., Vol. 11(1), R63, 2004.

[19] D. B. West. Introduction to graph theory. Prentice Hall, 1996. 